\title{
Learning-based Adaptive Control via Contraction Theory
}

\author{
Hiroyasu Tsukamoto*, Soon-Jo Chung*, and Jean-Jacques Slotine ${ }^{\dagger}$
}

\begin{abstract}
We present a new deep learning-based adaptive control framework for nonlinear systems with multiplicativelyseparable parametric uncertainty, called an adaptive Neural Contraction Metric (aNCM). The aNCM uses a neural network model of an optimal adaptive contraction metric, the existence of which guarantees asymptotic stability and exponential boundedness of system trajectories under the parametric uncertainty. In particular, we exploit the concept of a Neural Contraction Metric (NCM) to obtain a nominal provably stable robust control policy for nonlinear systems with bounded disturbances, and combine this policy with a novel adaptation law to achieve stability guarantees. We also show that the framework is applicable to adaptive control of dynamical systems modeled via basis function approximation. Furthermore, the use of neural networks in the aNCM permits its real-time implementation, resulting in broad applicability to a variety of systems. Its superiority to the state-of-the-art is illustrated with a simple cart-pole balancing task.
\end{abstract}

\section{INTRODUCTION}

Future aerospace and robotic exploration missions require that autonomous agents perform complex control tasks in extreme and unknown environments, while ensuring stability and optimality even for poorly modeled dynamical systems. Especially when the uncertainties are too large to be treated robustly as external disturbances, real-time implementable adaptive control schemes with provable stability certificates would enhance the autonomous capabilities of these agents.

In this work, we derive a method of adaptive Neural Contraction Metric (aNCM), which establishes a deep learningbased adaptive controller for nonlinear systems with parametric uncertainty. We consider multiplicatively-separable systems in terms of its state $x$ and unknown parameter $\theta$, i.e., $f(x, \theta)=Y(x)^{\top} Z(\theta)$, which holds for many types of systems including robotics systems [1], high-fidelity spacecraft dynamics [2], [3], and systems modeled by basis function approximation or neural networks [4], [5]. The aNCM is a real-time implementable neural network model of an optimal adaptive contraction metric, the existence of which guarantees global asymptotic stability and local exponential stability of system trajectories under such parametric uncertainty. It is constructed to minimize an upper bound of steady-state tracking error explicitly considering a target trajectory $x_{d}$ and estimated parameter $\hat{\theta}$ as the network inputs as in Fig. 1 unlike the Neural Contraction Metric (NCM) [6], where we proposed learning-based construction of optimal contraction

\footnotetext{
* Graduate Aerospace Laboratories, California Institute of Technology, Pasadena, CA, \{htsukamoto, sjchung\}@caltech.edu.

$\dagger$ Nonlinear Systems Laboratory, Massachusetts Institute of Technology, Cambridge MA, jjsamit.edu.

This work was in part funded by the Jet Propulsion Laboratory, California Institute of Technology. Code: https://github.com/astrohiro/ancm.
}

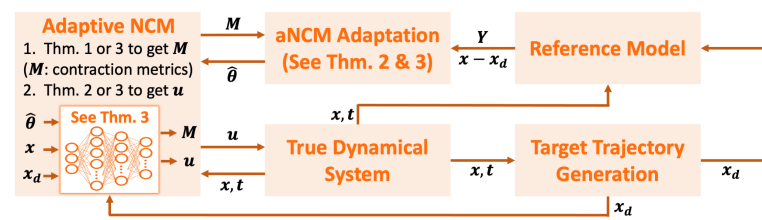

Fig. 1. Illustration of aNCM ( $M:$ aNCM; $\hat{\theta}$ : estimated parameter; $Y$ : error signal, see (17); $x(t)$ and $x_{d}(t)$ : actual and target state; $u$ : control input.

metrics for control and estimation of nonlinear systems with bounded disturbances. Asymptotic stability follows from a novel adaptation law expressed using the aNCM, and prior knowledge on the unknown parameter leads to exponential boundedness of system trajectories under this adaptation. The aNCM design in Fig. 1 and our contributions are as follows.

We first propose NCM-based adaptive control for systems with matched uncertainty, using the NCM as a nominal control for the sake of asymptotic and exponential stabilization. Its adaptation law exploits the generalized StateDependent Coefficient (SDC) parameterization (i.e. $A\left(x, x_{d}\right)$ s.t. $\left.A\left(x, x_{d}\right)\left(x-x_{d}\right)=f(x)-f\left(x_{d}\right)\right)$ [7] to guarantee stability even with a given target trajectory $\left(x_{d}, u_{d}\right)$, simplifying the differential formulation proposed in [8]. We further generalize this approach to multiplicatively-separable systems with $f(x, \theta)=Y(x)^{\top} Z(\theta)$ where $\theta$ is an unknown parameter, using the aNCM to model optimal contraction metrics as a function of both the system state $x$ and parameter estimate $\hat{\theta}$. The optimality of the aNCM follows from the ConVex optimization-based Steady-state Tracking Error Minimization (CV-STEM) [6], [7], [9], which constructs a contraction metric via convex optimization to minimize a steady-state upper bound of the tracking error under stochastic and deterministic disturbances. The aNCM approximates the solutions sampled by the CV-STEM at each state and parameter using a neural network along with the novel adaptation law built upon [1], [8] for asymptotic stabilization. We also present an important feature of the aNCM, i.e., its applicability to provably stable adaptive control of systems modeled by neural networks and basis function approximation [4], [5]. The proposed aNCM frameworks are demonstrated to outperform existing state-of-the-art adaptive and robust control techniques in the cart-pole balancing task in Fig. 2. We remark that the concept of implicit regularization-based adaptation [10] can also be incorporated in our framework to avoid overfitting issues due to over-parameterization.

Related Work: There exist well-developed adaptive stabilization techniques for nonlinear systems equipped with some special structures in their dynamics [1], [11]-[15]. They typically construct adaptive control schemes on top of a known Lyapunov function often found based on physical 
intuition (e.g. $V=q^{\top} H(q) q$ for robotics systems where $H(q)$ is an inertia matrix [1]). However, finding a Lyapunov function analytically without any prior knowledge of the systems of interest is challenging in general.

Developing numerical schemes for constructing a Lyapunov function in a given hypothesis function space has thus been an active field of research [16]-[19]. In particular, contraction analysis [20], where stability can be studied using a quadratic Lyapunov function of a differential state $\delta x$ (i.e. $V=\delta x^{\top} M(x) \delta x$ ) leads to convex optimization-based search algorithms for a contraction metric $M(x)$ [9], [21]-[23]. In [8], it is proposed that the obtained contraction metric can be exploited to estimate unknown system parameters adaptively with rigorous asymptotic stability guarantees, but its limitation lies in the fact that its problem size grows exponentially with the number of variables and basis functions [24].

We could also utilize over-parameterized mathematical models to approximate the true dynamical system and control laws with sampled data [6], [7], [25], [26]. This includes [27], where a spectrally-normalized neural network is used to model unknown residual dynamics with a nominal feedback linearization controller. When the modeling errors are sufficiently small with a large enough training dataset, these techniques yield promising control performance even for general cases with no prior knowledge on the underlying dynamical system. However, poorly modeled systems with insufficient training data result in conservative stability and robustness certificates [27], [28] unlike the aforementioned adaptive control techniques. Our proposed aNCM is for combining the provably stable adaptive control schemes via contraction theory, with the emerging machine learningbased techniques for broader applicability [6], [7].

Notation: For $x \in \mathbb{R}^{n}$ and $A \in \mathbb{R}^{n \times m}$, we let $\|x\|, \delta x$, and $\|A\|$, denote the Euclidean norm, infinitesimal variation of $x$, and induced 2-norm, respectively. For a square matrix $A$, we use the notation $A \succ 0, A \succeq 0, A \prec 0$, and $A \preceq 0$ for the positive definite, positive semi-definite, negative definite, negative semi-definite matrices, respectively, and $\operatorname{sym}(A)=$ $\left(A+A^{\top}\right) / 2$. Also, $I \in \mathbb{R}^{n \times n}$ denotes the identity matrix.

\section{Neural Contraction Metrics for Trajectory TRACKING CONTROL}

The Neural Contraction Metric (NCM) is a new deep learning-based design framework for provably stable feedback control of nonlinear systems given as $\dot{x}(t)=f(x(t))+$ $d(x)$, where $\bar{d}=\sup _{x}\|d(x)\|<+\infty$ [6]. This section presents its modified version for tracking control, which explicitly considers a given target trajectory $\left(x_{d}, u_{d}\right)$ governed by the following dynamical system with a controller $u \in \mathbb{R}^{m}$ :

$$
\dot{x}=f(x)+B(x) u+d(x), \dot{x}_{d}=f\left(x_{d}\right)+B\left(x_{d}\right) u_{d}\left(x_{d}\right)
$$

where $t \in \mathbb{R}_{\geq 0}, x, x_{d}: \mathbb{R}_{\geq 0} \mapsto \mathbb{R}^{n}, u_{d}: \mathbb{R}^{n} \mapsto \mathbb{R}^{n}, f: \mathbb{R}^{n} \mapsto \mathbb{R}^{n}$, and $B: \mathbb{R}^{n} \mapsto \mathbb{R}^{n \times m}$. Lemma 1 is useful for this purpose.

Lemma 1: Suppose $f$ and $B$ are piecewise continuously differentiable. Then $\forall x, x_{d}, \exists A\left(x, x_{d}\right)$ s.t. $f(x)+B(x) u_{d}\left(x_{d}\right)-$ $f\left(x_{d}\right)-B\left(x_{d}\right) u_{d}\left(x_{d}\right)=A\left(x, x_{d}\right)\left(x-x_{d}\right)$, and one such $A$ is given as $A\left(x, x_{d}\right)=\int_{0}^{1} \bar{f}_{x}\left(c x+(1-c) x_{d}\right) d c$, where $\bar{f}(q)=$ $f(q)+B(q) u_{d}\left(x_{d}\right)$. We call $A$ an SDC parameterization.

Proof: See [7].

Lemma 1 allows us to incorporate $\left(x_{d}, u_{d}\right)$ in the NCM formulation as follows.

Theorem 1: Let $B=B(x)$ and $A=A\left(x, x_{d}\right)$ be an SDC form of the dynamical system (1) given by Lemma 1 for notational simplicity. Consider a neural network trained to perfectly model the optimal contraction metric $M\left(x, x_{d}\right)=$ $W\left(x, x_{d}\right)^{-1} \succ 0$ (we call such $M$ a modified Neural Contraction Metric (NCM)), given by the following convex optimization problem:

$$
J_{C V}^{*}=\min _{\chi \in \mathbb{R}, \bar{W} \succ 0}(\bar{d} \chi / \alpha) \quad \text { s.t. (3) and (4). }
$$

with the convex constraints (3) and (4) given as

$$
\begin{aligned}
& -\dot{\bar{W}}+2 \operatorname{sym}(A \bar{W})-2 v B R^{-1} B^{\top} \preceq-2 \alpha \bar{W}, \forall x, x_{d} \\
& I \preceq \bar{W}\left(x, x_{d}\right) \preceq \chi I, \forall x, x_{d}
\end{aligned}
$$

where $\alpha, \underline{\omega}, \bar{\omega}>0, \chi=\bar{\omega} / \underline{\omega}, \bar{W}=v W, v=1 / \underline{\omega}$, and $R=$ $R\left(x, x_{d}\right) \succ 0$ is a given weight matrix on the control input $u$. If the dynamics (1) is controlled by

$$
u=u_{d}\left(x_{d}\right)-R\left(x, x_{d}\right)^{-1} B(x)^{\top} M\left(x, x_{d}\right)\left(x-x_{d}\right)
$$

then the Euclidean distance between $x$ and $x_{d}$ is exponentially bounded, and $M$ minimizes its steady-state upper bound.

Proof: The virtual system of (1) which has $x$ and $x_{d}$ as its particular solutions is given as $\dot{q}=\dot{x}_{d}+\left(A\left(x, x_{d}\right)-\right.$ $\left.B(x) R\left(x, x_{d}\right)^{-1} B(x)^{\top} M\left(x, x_{d}\right)\right)\left(q-x_{d}\right)+d_{q}$, where $d_{q}(x)=$ $d(x)$ and $d_{q}\left(x_{d}\right)=0$. Thus for a Lyapunov function $V=$ $\delta q^{\top} M \delta q$, we have $\dot{V} \leq-2 \alpha V+2 \delta q^{\top} M \delta d_{q}$ by construction of $A$ as in the proof of Theorem 2 in [7]. The rest follows from the proof of Corollary 1 in [6].

\section{Adaptive Neural Contraction Metrics}

In this section, we elucidate the method of adaptive Neural Contraction Metric (aNCM) depicted in Fig. 1 for designing real-time implementable control policies with an asymptotic stability guarantee.

\section{A. Affine Parameter Uncertainty}

We first consider the following dynamical systems:

$$
\begin{aligned}
\dot{x} & =f(x)+B(x) u-\Delta(x)^{\top} \theta \\
\dot{x}_{d} & =f\left(x_{d}\right)+B\left(x_{d}\right) u_{d}-\Delta\left(x_{d}\right)^{\top} \theta
\end{aligned}
$$

where $\theta \in \mathbb{R}^{p}, \Delta: \mathbb{R}^{n} \mapsto \mathbb{R}^{n \times p}$, and the other variables are as defined in (1). For these systems with the matched uncertainty condition [8], the modified NCM in Theorem 1 can be utilized to design its adaptive counterpart.

Theorem 2: Suppose that $f$ and $B$ in (6) are continuously differentiable, and we have $M\left(x, x_{d}\right)$ given by (2) in Theorem 1 for the nominal system (i.e. (6) and (7) with $\left.\Delta=0, \forall x, x_{d}\right)$. Suppose also that $\partial_{b_{i}(x)} M+\partial_{b_{i}\left(x_{d}\right)} M=0$, where $\partial_{v(q)} M=\sum_{i}\left(\partial M / \partial q_{i}\right) v_{i}$ and $B=\left[b_{1}, \cdots, b_{m}\right]$. If the matched uncertainty condition holds, i.e. $\left(\Delta(x)-\Delta\left(x_{d}\right)\right)^{\top} \theta \in$ $\operatorname{span}(B(x))$, and if there exists a bounded and Lipschitz function $\gamma\left(x, x_{d}\right)>0$ which satisfies (3) with equality when 
$\alpha$ is replaced by $\gamma\left(x, x_{d}\right)$, then the following adaptive control law guarantees asymptotic stability of (6):

$$
\begin{aligned}
& u=u_{d}-R\left(x, x_{d}\right)^{-1} B(x)^{\top} M\left(x, x_{d}\right)\left(x-x_{d}\right)+\varphi\left(x, x_{d}\right)^{\top} \hat{\theta} \\
& \dot{\hat{\theta}}=-\Gamma \varphi\left(x, x_{d}\right) B(x)^{\top} M\left(x, x_{d}\right)\left(x-x_{d}\right)
\end{aligned}
$$

where $\Gamma \succ 0$ and $\left(\Delta(x)-\Delta\left(x_{d}\right)\right)^{\top} \theta=B(x) \varphi\left(x, x_{d}\right)^{\top} \theta$.

Proof: Let $A_{c l}=A\left(x, x_{d}\right)-B(x) B(x)^{\top} M\left(x, x_{d}\right)$. The error dynamics of (6) and (7) for $e=x-x_{d}$ is given as $\dot{e}=A_{c l} e+B(x) \varphi\left(x, x_{d}\right)^{\top} \tilde{\theta}$, where the relation $(\Delta(x)-$ $\left.\Delta\left(x_{d}\right)\right)^{\top} \theta=B(x) \varphi\left(x, x_{d}\right)^{\top} \theta$ is used. Thus for a Lyapunov function defined as $V=e^{\top} M\left(x, x_{d}\right) e+\tilde{\theta}^{\top} \Gamma^{-1} \tilde{\theta}$, we have $\dot{V} \leq-2 \alpha e^{\top} M e+2 e^{\top} M B \varphi^{\top} \tilde{\theta}+2 \tilde{\theta}^{\top} \Gamma^{-1} \dot{\tilde{\theta}}$ by the proof of Theorem 1 along with the relation $\partial_{b_{i}(x)} M+\partial_{b_{i}\left(x_{d}\right)} M=0$. Therefore, (9) gives $\dot{V} \leq-2 \alpha e^{\top} M e$, which implies boundedness of $x$ and $\hat{\theta}$ as $x_{d}$ and $\theta$ are bounded. This also gives bounded $\dot{x}$ and $\dot{x}_{d}$ due to (6) and (7). Since $\exists \gamma\left(x, x_{d}\right)>0$ s.t. $\dot{V}=-2 \gamma\left(x, x_{d}\right) e^{\top} M e$, we have $\ddot{V}=-2 \dot{\gamma} e^{\top} M e-4 \gamma e^{\top} M \dot{e}-$ $2 \gamma e^{\top} \dot{M} e$, which is bounded as its arguments are all bounded. Asymptotic convergence of $e$ to 0 then follows from Barbalat's lemma [1], [29]. An exponential stability result of $e$ can also be obtained with a boundeness assumption on $\tilde{\theta}$.

Corollary 1: The controller (8) with the adaptation law

$$
\dot{\hat{\theta}}=-\Gamma \varphi\left(x, x_{d}\right) B(x)^{\top} M\left(x, x_{d}\right)\left(x-x_{d}\right)-\alpha\left(\hat{\theta}-\theta_{n}\right)
$$

where $\theta_{n}$ is a guess for the true parameter $\theta$, gives the following exponential bound on the tracking error:

$$
\sqrt{\underline{\rho}}\left\|x-x_{d}\right\| \leq \sqrt{V\left(x(0), x_{d}(0)\right)} e^{-\alpha t}+\sqrt{\bar{\rho}}\left\|\theta-\theta_{n}\right\| .
$$

where $\rho, \bar{\rho}>0$ with $\rho I \preceq \operatorname{diag}\left(M, \Gamma^{-1}\right) \preceq \bar{\rho} I$.

Proof: For $V=e^{\top} M\left(x, x_{d}\right) e+\tilde{\theta}^{\top} \Gamma^{-1} \tilde{\theta}$, we have $\dot{V} \leq$ $-2 \alpha V+2 \alpha \tilde{\theta}^{\top} \Gamma^{-1}\left(\theta_{n}-\theta\right)$ by Theorem 2 , and thus $\dot{\mathscr{R}} \leq$ $-\alpha \mathscr{R}(t)+\alpha \sqrt{\bar{\rho}}\left\|\theta_{n}-\theta\right\|$, for $\mathscr{R}(t)=\left\|\Theta\left(x(t), x_{d}(t)\right) q(t)\right\|$ with $q=\left[e^{\top}, \tilde{\theta}^{\top}\right]^{\top}$ and $\operatorname{diag}\left(M, \Gamma^{-1}\right)=\Theta^{\top} \Theta$. Applying the comparison lemma [29] results in (11).

\section{B. Modified NCM for Lagrangian-type Nonlinear Systems}

We have thus far examined the case where $f(x)$ is affine in its parameter. This section considers the following dynamical system with an uncertain parameter $\theta$ and a control input $\tau$ :

$$
H(s) \dot{s}+h(s)+\Delta(s) \theta=\tau
$$

where $s \in \mathbb{R}^{n}, \tau \in \mathbb{R}^{n}, H: \mathbb{R}^{n} \mapsto \mathbb{R}^{n \times n}, h: \mathbb{R}^{n} \mapsto \mathbb{R}^{n}, \Delta: \mathbb{R}^{n} \mapsto$ $\mathbb{R}^{n \times p}$, and $H(s)$ is non-singular for all $s$. We often encounter the problem of designing a controller with an asymptotic convergence guarantee, $s \rightarrow 0$, one example of which is the tracking control of Lagrangian systems [1]. The modified NCM is also applicable to such problems.

Corollary 2: Let $M(s)$ be an NCM for the dynamical system $\dot{s}=-H(s)^{-1} h(s)+H(s)^{-1} \tau$ given by (2) of Theorem 1, which satisfies $\partial_{b_{i}(s)} M=0$ for $\partial_{v(q)} M=\sum_{i}\left(\partial M / \partial q_{i}\right) v_{i}$ and $B(s)=H(s)^{-1}=\left[b_{1}, \cdots, b_{m}\right]$. If we design $\tau$ as

$$
\tau=-R(s)^{-1} H(s)^{-\top} M s+\Delta(s) \hat{\theta}, \dot{\hat{\theta}}=-\Gamma \Delta(s)^{\top} H(s)^{-\top} M s
$$

where $R(s) \succ 0$ is a given weight matrix on $\tau$, and if there exists a bounded and Lipschitz function $\gamma\left(x, x_{d}\right)>0$ which satisfies (3) with equality when $\alpha$ is replaced by $\gamma\left(x, x_{d}\right)$, then $s$ of (12) asymptotically converges to 0 .

Proof: We have for $V=s^{\top} M s+\tilde{\theta}^{\top} \Gamma^{-1} \tilde{\theta}$ that $\dot{V} / 2 \leq$ $-\alpha s^{\top} M s+s^{\top} M H^{-1} \Delta \tilde{\theta}+\tilde{\theta}^{\top} \Gamma^{-1} \dot{\tilde{\theta}}$ using $\partial_{b_{i}(s)} M=0$. Thus, (13) gives $\dot{V} \leq-2 \alpha s^{\top} M s$, which results in asymptotic stability by the proof of Theorem 2 .

Remark 1: An exponential boundedness result as in Corollary 1 is obtainable with $\dot{\hat{\theta}}=-\Gamma \Delta(s)^{\top} H(s)^{-\top} M(s) s-$ $2 \alpha\left(\hat{\theta}-\theta_{n}\right)$. Also, the Lipschitz condition on $\gamma$ (or on $M$ ) can be guaranteed by using spectral normalization [7], [27].

\section{Multiplicatively-Separable Parameter Uncertainty}

Next, let us consider the following nonlinear system with an uncertain parameter $\theta \in \mathbb{R}^{p}$ :

$$
\dot{x}=f(x ; \theta)+B(x ; \theta) u, \dot{x}_{d}=f\left(x_{d} ; \theta\right)+B\left(x_{d} ; \theta\right) u_{d} .
$$

In this section, we assume the following.

Assumption 1: The system (22) is multiplicativelyseparable in terms of $x$ and $\theta$, i.e., $\exists Y_{f}: \mathbb{R}^{n} \mapsto \mathbb{R}^{n \times q_{z}}$, $Y_{b_{i}}: \mathbb{R}^{n} \mapsto \mathbb{R}^{n \times q_{z}}, \forall i$, and $Z: \mathbb{R}^{p} \mapsto \mathbb{R}^{q_{z}}$ s.t.

$$
Y_{f}(x) Z(\theta)=f(x ; \theta), Y_{b_{i}}(x) Z(\theta)=b_{i}(x ; \theta), \forall x, \theta
$$

where $B(x ; \theta)=\left[b_{1}(x ; \theta), \cdots, b_{m}(x ; \theta)\right]$.

Remark 2: When Assumption 1 holds, we could always redefine $\theta$ as $\left[\theta^{\top}, Z(\theta)^{\top}\right]^{\top}$ to have $Y_{f}(q) \theta=f(q ; \theta)$ and $Y_{b_{i}}(q) \theta=b_{i}(q ; \theta)$. Since we can implicitly regularize such an over-parameterized system using the Bregman divergence as will be seen in Corollary 3 , we denote $\left[\theta^{\top}, Z(\theta)^{\top}\right]^{\top}$ as $\theta$ in the subsequent discussion.

Under Assumption 1 with $\theta$ augmented as $\left[\theta^{\top}, Z(\theta)^{\top}\right]^{\top}$, the dynamics for $e=x-x_{d}$ of (14) is expressed as follows:

$$
\begin{aligned}
\dot{e} & =A\left(x, x_{d} ; \hat{\theta}\right) e+B(x ; \hat{\theta})\left(u-u_{d}\right)-\tilde{Y}(\hat{\theta}-\theta) \\
\tilde{Y} & =Y-Y_{d}=\left(Y_{f}(x)+Y_{b}(x, u)\right)-\left(Y_{f}\left(x_{d}\right)+Y_{b}\left(x_{d}, u_{d}\right)\right)
\end{aligned}
$$

where $Y_{b}(x, u)=\sum_{i=1}^{m} Y_{b_{i}}(q) u_{i}, A$ is the SDC matrix defined in Lemma 1 , and $\hat{\theta}$ is the current estimate of $\theta$. The following lemma is useful for stability analysis of (16).

Lemma 2: For a Lyapunov function $V\left(x, x_{d}\right)=R\left(x, x_{d}\right)^{2}$ where $R$ is continuously differentiable, $\exists M\left(x, x_{d}\right)$ s.t. $V\left(x, x_{d}\right)=e^{\top} M\left(x, x_{d}\right) e$.

Proof: As in Lemma 1, $\exists \mu$ s.t. $R=\mu\left(x-x_{d}\right)=$ $\left(\int_{\underline{0}}^{1} R_{x}\left(c x+(1-c) x_{d}, x_{d}\right) d c\right)\left(x-x_{d}\right)$. Defining $M$ as $M=$ $\mu^{\top} \mu$ gives the desired relation.

Lemma 2 indicates that the problem of finding a Lyapunov function $V\left(x, x_{d}\right)$ reduces to the problem of finding a metric $M\left(x, x_{d}\right)$, leading to the following theorem on the NCMbased adaptive control for asymptotic stabilization of (16).

Theorem 3: Suppose that Assumption 1 holds and let $B=B(x ; \hat{\theta})$ and $A=A\left(x, x_{d} ; \hat{\theta}\right)$ be the SDC matrix given in (16) for notational simplicity. Consider a neural network trained to perfectly model the optimal contraction metric $M\left(x, x_{d}, \hat{\theta}\right)=W\left(x, x_{d}, \hat{\theta}\right)^{-1}$ (we call such $M$ an adaptive Neural Contraction Metric (aNCM)), given by the following convex optimization problem:

$$
J_{a C V}^{*}=\min _{\chi \in \mathbb{R}, \bar{W} \succ 0}(\bar{d} \chi / \alpha) \text { s.t. (19) and (20). }
$$


with the convex constraints (19) and (20) given as

$$
\begin{aligned}
& -\left.(d / d t)\right|_{\hat{\theta}} \bar{W}+2 \operatorname{sym}(A \bar{W})-2 v B R^{-1} B^{\top} \preceq-2 \alpha \bar{W} \\
& I \preceq \bar{W}\left(x, x_{d}, \hat{\theta}\right) \preceq \chi I, \forall x, x_{d}, \hat{\theta}
\end{aligned}
$$

where $\alpha, \underline{\omega}, \bar{\omega}>0, \chi=\bar{\omega} / \underline{\omega}, \bar{W}=v W, v=1 / \underline{\omega},\left.(d / d t)\right|_{\hat{\theta}} \bar{W}$ is the time derivative of $\bar{W}$ computed along (14) with $\theta=\hat{\theta}$, and $R=R\left(x, x_{d}\right) \succ 0$ is a given weight matrix on $u$. If the true dynamics (14) is controlled by

$$
\begin{aligned}
& u=u_{d}-R\left(x, x_{d}\right)^{-1} B(x ; \hat{\theta})^{\top} M\left(x, x_{d}, \hat{\theta}\right)\left(x-x_{d}\right) \\
& \dot{\hat{\theta}}=\Gamma\left(Y^{\top} d M_{x}+Y_{d}^{\top} d M_{x_{d}}+\tilde{Y}^{\top} M\right)\left(x-x_{d}\right)
\end{aligned}
$$

where $d M_{q}=\left[\left(\partial M / \partial q_{1}\right) e, \cdots,\left(\partial M / \partial q_{n}\right) e\right]^{\top} / 2, e=x-x_{d}$, $\Gamma \succ 0$, and $Y, Y_{d}, \tilde{Y}$ are as defined in (17), and if there exists a bounded and Lipschitz function $\gamma\left(x, x_{d}\right)>0$ which satisfies (19) with equality when $\alpha$ is replaced by $\gamma\left(x, x_{d}\right)$, then $e$ of (16) asymptotically converges to 0 . Also, when $\theta$ in (14) is given, the Euclidean distance between $x_{d}$ and $x$ perturbed by bounded disturbances is exponentially bounded, and the metric $M$ minimizes its steady-state upper bound.

Proof: Computing $\dot{M} e$ along the dynamics (14) yields $\dot{M} e=\left(\left.(d / d t)\right|_{\hat{\theta}} M\right) e-2\left(d M_{x}^{\top} Y+d M_{x_{d}}^{\top} Y_{d}\right) \tilde{\theta}$, where $\left.(d / d t)\right|_{\hat{\theta}} M$ is the time derivative of $M$ computed along (14) with $\theta=\hat{\theta}$. Thus for $V=e^{\top} M e+\tilde{\theta}^{\top} \Gamma^{-1} \tilde{\theta}$, we have $\dot{V} \leq$ $-2 \alpha e^{\top} M e-2 e^{\top}\left(d M_{x}^{\top} Y+d M_{x_{d}}^{\top} Y_{d}+M \tilde{Y}\right) \tilde{\theta}+\tilde{\theta}^{\top} \Gamma \dot{\tilde{\theta}}$ by (16), (21), and Theorem 1 along with (19) and (20). The adaptation law (22) applied to this relation gives $\dot{V} \leq-2 \alpha e^{\top} M e$, which implies asymptotic stability of (16) as proved in Theorem 2 using Barbalat's lemma. The final statement on optimality of $M$ follows by Theorem 1. Exponential stability of $e$ can also be obtained with a boundeness assumption on $\tilde{\theta}$.

We could also incorporate the Bregman divergence-based implicit regularization on its parameter convergence [10].

Corollary 3: Let $\psi: \mathbb{R}^{p} \mapsto \mathbb{R}$ be a given convex function and consider an adaptive controller (21) for (22) with the following adaptation law

$$
\dot{\hat{\theta}}=\left(\nabla^{2} \psi(\hat{\theta})\right)^{-1}\left(Y^{\top} d M_{x}+Y_{d}^{\top} d M_{x_{d}}+\tilde{Y}^{\top} M\right)\left(x-x_{d}\right) .
$$

Then $e$ of (16) asymptotically converges to 0 under the same assumptions as in Theorem 3. Also, suppose that $\theta^{*}=\lim _{t \rightarrow \infty} \hat{\theta}(t) \in A$, where $A=\left\{\vartheta \mid \Xi(t)^{\top} \vartheta=\Xi(t)^{\top} \theta, \forall t\right\}$ for the true parameter $\theta$ with $\Xi(\tau)=\left(Y^{\top} d M_{x}+Y_{d}^{\top} d M_{x_{d}}+\right.$ $\left.\tilde{Y}^{\top} M\right)\left.\right|_{t=\tau}$. Then we have

$$
\theta^{*}=\arg \min _{\vartheta \in A} d_{\psi}\left(\vartheta \| \theta^{*}\right)=\arg \min _{\vartheta \in A} d_{\psi}(\vartheta \| \hat{\theta}(0))
$$

where $d_{\psi}$ is the Bregman divergence defined as $d_{\psi}(x \| y)=$ $\psi(x)-\psi(y)-(x-y)^{\top} \nabla \psi(y)$. Further, selecting $\hat{\theta}(0)$ as $\hat{\theta}(0)=\arg \min _{b \in \mathbb{R}^{p}} \psi(b)$ yields $\theta^{*}=\arg \min _{\vartheta \in A} \psi(\vartheta)$.

Proof: Asymptotic stability follows by the proof of Theorem 2 applied to $V=e^{\top} M e+2 d_{\psi}(\theta \mid \hat{\theta})$ with (23) and with the property of the Bregman divergence $d\left(d_{\psi}(\theta \| \hat{\theta})\right) / d t=(\hat{\theta}-\theta)^{\top} \nabla^{2} \psi(\hat{\theta}) \dot{\hat{\theta}}$. Next, we have for any $\vartheta \in A=\left\{\vartheta \mid \Xi(t)^{\top} \vartheta=\Xi(t)^{\top} \theta, \forall t\right\}$ that $d_{\psi}\left(\vartheta \| \theta^{*}\right)-$ $d_{\psi}(\vartheta \| \hat{\theta}(0))=\int_{0}^{\infty}(\hat{\theta}(\tau)-\theta)^{\top} \Xi(\tau) e(\tau) d \tau$, where $\Xi(\tau)=$ $\left.\left(Y^{\top} d M_{x}+Y_{d}^{\top} d M_{x_{d}}+\tilde{Y}^{\top} M\right)\right|_{t=\tau}$. Since the right-hand side does not depend on $\vartheta$ and its minimum over $\vartheta \in A$ is clearly obtained at $\theta^{*} \in A$, we have (24) as desired. Also, $\hat{\theta}(0)=$ $\arg \min _{b \in \mathbb{R}^{p}} \psi(b)$ implies $\nabla \psi(\hat{\theta}(0))=0$ by the convexity of $\psi$, resulting in $d_{\psi}(\vartheta \| \hat{\theta}(0))=\psi(\vartheta)$.

Remark 3: An important example of $\psi$ in (3) is a norm, which could regularize the unknown parameter implicitly by (24). Using different types of norms leads to different properties of the steady-state estimated parameter such as sparsity [10].

The following corollary could be more useful than Theorem 3 or Corollary 3 in practical applications.

Corollary 4: Adding the decaying term $-2 \alpha\left(\hat{\theta}-\theta_{n}\right)$ with the nominal parameter $\theta_{n}$ to the adaptation laws (22) and (23) leads to exponential boundedness of the tacking error.

Proof: See Corollary 1.

Remark 4: We could remove the dependence on $u$ and $\dot{\theta}$ in $\left.(d / d t)\right|_{\hat{\theta}} M$ by incorporating $\partial_{b_{i}(x)} M+\partial_{b_{i}\left(x_{d}\right)} M=0$ and by using the extended matched uncertainty condition [8], [22].

\section{Practical Application of ANCM Control}

This section delineates how to apply the aNCM control law to several important engineering and robotics examples, and proposes practical numerical algorithms to construct aNCMs using Theorem 2 and 3.

\section{A. Systems Modeled by Function Approximators}

Utilization of function approximators, neural networks in particular, has gained great popularity in system identification due to their high representational power, and provably stable techniques for using these approximators in closedloop have been derived in [5]. The aNCM framework can also be used to guarantee asymptotic stability and exponential boundedness of system trajectories in this context.

Consider the following system with basis functions $\phi(x)=\left[\phi_{1}(x), \cdots, \phi_{p}(x)\right]^{\top}$ and $\varphi_{i}(x)=\left[\varphi_{i, 1}(x), \cdots, \varphi_{i, q}(x)\right]^{\top}$, $i=1, \cdots, m$, which approximate $f(x)$ and $B(x)=$ $\left[b_{1}(x), \cdots, b_{m}(x)\right]$ of the true dynamical system, respectively:

$$
\dot{x}=f(x)+B(x) u \simeq \mathscr{F} \phi(x)+\sum_{i=1}^{m} \mathscr{B}_{i} \varphi_{i}(x) u_{i}
$$

where $\mathscr{F} \in \mathbb{R}^{n \times p}, \mathscr{B}_{i} \in \mathbb{R}^{n \times q}, \mathscr{F} \phi(x) \simeq f(x)$, and $\mathscr{B}_{i} \varphi_{i}(x) \simeq$ $b_{i}(x)$. The following proposition introduces aNCM-based adaptation laws to update the weights $\mathscr{F}$ and $\mathscr{B}_{i}$ for asymptotic stabilization.

Proposition 1: Suppose $\exists \mathscr{F}^{*}$ and $\mathscr{B}_{i}^{*}$ s.t. $\mathscr{F}^{*} \phi(x)=f(x)$ and $\mathscr{B}_{i}^{*} \varphi_{i}(x)=b_{i}(x), \forall i, \forall x \in \mathbb{R}^{n}$. Let $M\left(x, x_{d}, \hat{\mathscr{F}}, \hat{\mathscr{B}}\right)$ be the aNCM for (25) given by (18) with the same assumptions of Theorem 3, where $\hat{\mathscr{F}}$ and $\hat{\mathscr{B}}$ are the estimates of $\mathscr{F}^{*}$ and $\mathscr{B}^{*}$. Also, let $\mathscr{W}$ denote the weights, and let $\zeta=\phi(x)$ and $\zeta_{d}=\phi\left(x_{d}\right)$ for $\mathscr{W}=\mathscr{F}$, and $\zeta=\varphi_{i}(x) u_{i}$ and $\zeta_{d}=\varphi_{i}\left(x_{d}\right) u_{d, i}$ for $\mathscr{W}=\mathscr{B}_{i}$. If $u$ is given by (21), the following adaptation

$$
\dot{\hat{\mathscr{W}}}=\left(\nabla^{2} \psi(\hat{\mathscr{W}})\right)^{-1}:\left(d M_{x} e \zeta^{\top}+d M_{x_{d}} e \zeta_{d}^{\top}+M e \tilde{\zeta}^{\top}\right)
$$

where $\tilde{\zeta}=\zeta-\zeta_{d}$ and $:$ is defined as $(A: B)_{i j}=\sum_{k, \ell} A_{i j k \ell} B_{\ell k}$, guarantees asymptotic convergence of $e=x-x_{d}$ to 0 for (25). Note that $\nabla^{2} \psi(\cdot)$ represents the fourth order tensor given as $\nabla^{2} \psi(\cdot)_{i j k \ell}=\partial^{2} \psi /\left(\partial(\cdot)_{i j} \partial(\cdot)_{\ell k}\right)$ for a convex function $\psi(\cdot)$. Further, $\hat{\mathscr{F}}$ and $\hat{\mathscr{B}}_{i}$ are implicitly regularized by $\psi$ when 
they converge to the true parameter in the sets $\left\{\vartheta \mid M\left(\mathscr{W}^{*}-\right.\right.$ $\left.\vartheta) \tilde{\zeta}+d M_{x}^{\top}\left(\mathscr{W}^{*}-\vartheta\right) \zeta+d M_{x_{d}}^{\top}\left(\mathscr{W}^{*}-\vartheta\right) \zeta_{d}=0\right\}$. Adding the decaying term to (26) leads to exponential boundedness of the tracking error as in Corollary 4.

Proof: Let us define $V$ as $V=2 \sum_{\mathscr{W}=\mathscr{F}, \mathscr{B}_{i}} d_{\psi}\left(\mathscr{W}^{*} \mid \hat{\mathscr{W}}\right)+$ $e^{\top} M e$, where $d_{\psi}(\theta \| \hat{\theta})=\psi(\theta)-\psi(\hat{\theta})+(\hat{\theta}-\theta):(\nabla \psi(\hat{\theta}))$. Also, let $V_{e}=e^{\top} M e$ and $\tilde{\mathscr{W}}=\mathscr{\mathscr { W }}-\mathscr{W}^{*}$. Since $M$ is the aNCM of (19), we have as in the proof of Theorem 3 that $\dot{V}_{e} \leq-2 \alpha e^{\top} M e-2 e^{\top} \sum_{\mathscr{W}=\mathscr{F}, \mathscr{B}_{i}}\left(d M_{x}^{\top} \tilde{\mathscr{W}} \zeta+d M_{x_{d}}^{\top} \tilde{\mathscr{W}} \zeta_{d}+\right.$ $M \tilde{\mathscr{W}} \tilde{\zeta})$. This along with (26) gives $\dot{V} \leq-2 \alpha e^{\top} M e$ due to $a^{\top} C b=C:\left(a b^{\top}\right)$ for $a \in \mathbb{R}^{n}, b \in \mathbb{R}^{p}$, and $C \in \mathbb{R}^{n \times p}$. The regularization statement follows from Corollary 3.

We can utilize the same technique for neural net modeled systems to adaptively update the weights of its last layer.

Corollary 5: Consider the following system, $\dot{x}=f(x)+$ $B(x) u=\mathscr{F}^{*} \rho\left(x ; \mathscr{F}_{1: L}\right)+\sum_{i=1}^{m} \mathscr{B}_{i}^{*} \beta_{i}\left(x ; \mathscr{B}_{1: L}^{(i)}\right) u_{i}$, where $\rho$ and $\beta_{i}$ are the nominal neural nets with the trained weights of the first $L$ layers, $\overline{\mathscr{F}}_{1: L}$ and $\overline{\mathscr{B}}_{1: L}^{(i)}$, and $\mathscr{F}^{*}$ and $\mathscr{B}_{i}^{*}$ are the true weights of their last layers. Then the aNCM control (21) under the adaptation law (26) with $\phi(x)$ and $\varphi_{i}(x)$ replaced by $\rho\left(x ; \mathscr{F}_{1: L}\right)$ and $\beta_{i}\left(x ; \mathscr{B}_{1: L}^{(i)}\right)$, respectively, guarantees asymptotic convergence of $e=x-x_{d}$ to 0 .

Proof: See the proof of Proposition 1.

\section{B. Additional Remarks in aNCM Implementation}

We finally propose several useful implementation techniques for the practical application of the aNCM framework introduced in Theorem 2 and 3.

1) Backward Approximation of Time Derivatives: The construction of $M$ by (2) and (18) requires computation of $\dot{W}$ and $\left.(d / d t)\right|_{\hat{\theta}} W$, respectively. One way to solve them as a finite-dimensional problem is to apply $\dot{\tilde{W}}\left(x\left(t_{i}\right), t_{i}\right) \simeq$ $\left(\tilde{W}\left(x\left(t_{i}\right), t_{i}\right)-\tilde{W}\left(x\left(t_{i-1}\right), t_{i-1}\right)\right) / \Delta t_{i}$, where $\Delta t=t_{i}-t_{i-1}, \forall i$ with $\Delta t \gg \Delta t^{2}>0$ by using backward difference approximation on $\dot{\bar{W}}$, where we can then use $-\bar{W} \preceq-I$ to obtain a sufficient condition of its constraints. We could also solve it along pre-computed trajectories (see [6], [7], [30] for details).

2) Constraints as Loss Functions: Instead of solving (2) and (18) for $\bar{W}$ to sample training data $\left\{\left(x, x_{d}, M\right)\right\}_{i=1}^{N}$, we could directly solve them for the neural net weights treating the constraints as loss functions for stochastic gradient descent in the network training. Since it is challenging to optimize the weights from scratch, we could initialize them with the solution given by the NCM [6], and use spectral normalization [7], [27], [31] to regularize the network for bounding $\partial M / \partial q$ (i.e. $M$ : Lipschitz), where $q=x, x_{d}, \hat{\theta}$.

3) aNCMs with Control Lyapunov Functions: The aNCM can also be utilized as a Control Lyapunov Function (CLF) [32]. In particular, we consider a controller $u=$ $u_{d}+K^{*}\left(x, x_{d}\right) e$ in (14), where $K^{*}$ is given by

$$
\begin{aligned}
& K^{*}=\arg \min _{K \in \mathbb{R}^{m \times n}}\left\|u-u_{d}\right\|^{2}=\arg \min _{K \in \mathbb{R}^{m \times n}}\left\|K\left(x, x_{d}\right) e\right\|^{2} \\
& \text { s.t. }\left.(d / d t)\right|_{\hat{\theta}} M+2 \operatorname{sym}\left(M A+M K\left(x, x_{d}\right)\right) \leq-2 \alpha M .
\end{aligned}
$$

which is convex when $\left(x, x_{d}\right)$ is given at time $t$.

Proposition 2: If $M$ of (18) in Theorem 3 is perfectly modeled by a neural net, (27) is always feasible, i.e., $V=$

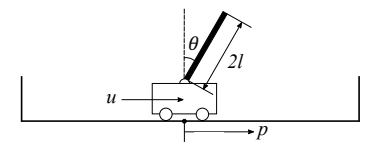

Fig. 2. Cart-pole balancing task.
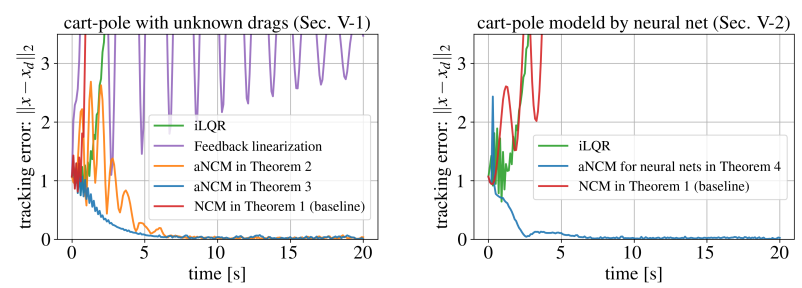

Fig. 3. Simulation results for cart-pole balancing task with unknown drags (LHS) and unknown dynamical system (RHS).

$e^{\top} M e$ is a CLF for $e=x-x_{d}$, and minimizes the deviation of $u$ from $u_{d}$ under the stability constraint (28). Further, $u=u_{d}+K^{*} e$ guarantees asymptotic stabilization of $e$ to 0 .

Proof: See [32].

Remark 5: For not perfectly modeled $M$, the optimizationbased controller (27) can be modified to guarantee its feasibility by rewriting the objective as $\|K e\|^{2}+p^{2}$ and (28) as $\left.(d / d t)\right|_{\hat{\theta}} M+2 \operatorname{sym}(M A+M K) \leq-2 \alpha M+p$, where $p$ is a decision variable to relax the stability constraint (28). Convex input constraints can be incorporated by using the same relaxation technique.

\section{Simulation}

We demonstrate the aNCM framework on a cart-pole balancing problem [33] (https://github.com/astrohiro/ancm). CVXPY [34] with the MOSEK solver [35] is used to solve convex optimization. The task is selected to drive the states $x=[p, \theta, \dot{p}, \dot{\theta}]^{\top}$ depicted in Fig. 2 to 0 controlling the following under-actuated dynamics, $\left(m_{c}+\right.$ $m) \ddot{p}+m l \cos \theta \ddot{\theta}=m l \dot{\theta}^{2} \sin \theta-\mu_{c} \dot{p}+u$, and $m l \cos \theta \ddot{p}+$ $(4 / 3) m l^{2} \ddot{\theta}=m l g \sin \theta-\mu_{p} \dot{\theta}$, where we used $g=9.8, m_{c}=$ $1.0, m=0.1, \mu_{c}=0.5, \mu_{p}=0.002$, and $l=0.5$. Note that the system with all the control laws in this section is perturbed by the same realization of the disturbance $d(x)$ with $\sup \|d\|=0.15$.

1) Cart-Pole Balancing with Unknown Drags: We first consider the case where the drag coefficients, $\mu_{c}$ and $\mu_{p}$, are unknown. Since this system satisfies Assumption 1, we can apply the aNCM in Theorem 3 for asymptotic stabilization. Also, although the uncertainty does not satisfy the matching condition in Theorem 2, a nominal NCM in Theorem 1 with the adaptation law (9) is implemented using the pseudoinverse of $B(x)$ in (6). Note that the adaptive robot trajectory control [1, pp. 403] for fully actuated Lagrangian systems is not applicable as the dynamics is under-actuated. We thus use it for partial feedback linearization as in (68) of [9]. We implement these three controllers to compare their performance with the iterative LQR (iLQR) [36] and baseline robust NCM control in Theorem 1 without any adaptation. The initial conditions are selected as $x(0)=[0.83,-0.32,0.39,0.45]^{\top}$, $\hat{\mu}_{c}(0)=4.0$, and $\hat{\mu}_{p}(0)=0.0016$. 
As can be seen from Fig. 3, the aNCM control law of Theorem 2 and 3 achieve asymptotic stabilization, while the other three baselines in [36], [1, pp. 403], and [6] fail to balance the pole. Also, the aNCM of Theorem 3 has better transient behavior than that of Theorem 2 as the matched uncertainty condition does not hold in this case.

2) Cart-Pole Balancing with Unknown Dynamical System: We next consider the case where the structure of the cartpole dynamics is completely unknown and thus modeled by a neural network using a limited amount of its trajectory data. The purpose of this section is to demonstrate the strength of the aNCM framework, which provides an asymptotic stability guarantee even for such systems by Proposition 1 . The number of neural net layers and hidden units are selected as 3 and 5, and we compare the performance of the aNCM control in Corollary 5 for updating the weight of the last layer, with that of the iLQR [36] and baseline robust NCM control in Theorem 1 constructed for the nominal neural net dynamical system model trained with 10000 data points.

As shown in the right-hand side of Fig. 3, the proposed aNCM control indeed achieves asymptotic stabilization even though the underlying dynamics is unknown, while the trajectories of the $\mathrm{iLQR}$ and robust NCM designed for the nominal neural net dynamical system diverge.

\section{CONCLUSION}

This work presents the method of aNCM, which models an optimal adaptive contraction metric to provide provable asymptotic stability for nonlinear systems with multiplicatively-separable parameter uncertainty. In essence, the neural network-based differential Lyapunov function via NCM guarantees stability of a nonlinear adaptive control law. This framework is shown to be applicable to wide range of systems including systems modeled by neural networks, and demonstrated to outperform existing state-of-the-art robust and adaptive control through numerical simulations. The aNCM can also be used for adaptive control of stochastic systems using the stochastic NCM [7], and to adaptive motion planning using the contraction-based imitation learning [32].

\section{REFERENCES}

[1] J.-J. E. Slotine and W. Li, Applied Nonlinear Control. Upper Saddle River, NJ: Pearson, 1991.

[2] R. H. Battin, An Introduction to the Mathematics and Methods of Astrodynamics. AIAA Education Series, 1999.

[3] D. Morgan, S.-J. Chung, L. Blackmore, B. Acikmese, D. Bayard, and F. Y. Hadaegh, "Swarm-keeping strategies for spacecraft under J2 and atmospheric drag perturbations," Journal of Guidance, Control, and Dynamics, vol. 35, no. 5, pp. 1492-1506, 2012.

[4] O. Nelles, Nonlinear Dynamic System Identification. Springer Berlin Heidelberg, 2001.

[5] R. M. Sanner and J.-J. E. Slotine, "Gaussian networks for direct adaptive control," vol. 3, no. 6, pp. 837-863, 1992.

[6] H. Tsukamoto and S.-J. Chung, "Neural contraction metrics for robust estimation and control: A convex optimization approach," IEEE Control Syst. Lett., vol. 5, no. 1, pp. 211-216, 2021.

[7] H. Tsukamoto, S.-J. Chung, and J.-J. E. Slotine, "Neural stochastic contraction metrics for robust control and estimation," Minor revision requested in IEEE Control Syst. Lett., 2020.

[8] B. T. Lopez and J.-J. E. Slotine, "Adaptive nonlinear control with contraction metrics," IEEE Control Systems Letters, vol. 5, no. 1, pp. 205-210, 2021.
[9] H. Tsukamoto and S.-J. Chung, "Robust controller design for stochastic nonlinear systems via convex optimization," IEEE Trans. Autom. Control, to appear, Oct. 2021.

[10] N. M. Boffi and J.-J. E. Slotine, "Implicit regularization and momentum algorithms in nonlinear adaptive control and prediction," arXiv:1912.13154, 2020.

[11] D. G. Taylor, P. V. Kokotovic, R. Marino, and I. Kannellakopoulos, "Adaptive regulation of nonlinear systems with unmodeled dynamics," IEEE Trans Autom. Control, vol. 34, no. 4, pp. 405-412, 1989.

[12] "Robustness of adaptive nonlinear control under an extended matching condition," IFAC Proc. Volumes, vol. 22, no. 3, pp. 245 - 250, 1989.

[13] G. Ambrosino, G. Celektano, and F. Garofalo, "Variable structure model reference adaptive control systems," Int. J. Control, vol. 39, no. 6 , pp. 1339-1349, 1984.

[14] J.-J. E. Slotine and J. A. Coetsee, "Adaptive sliding controller synthesis for non-linear systems," Int. J. Control, vol. 43, no. 6, pp. 16311651,1986

[15] M. Krstić, I. Kanellakopoulos, and P. Kokotović, "Adaptive nonlinear control without overparametrization," Syst. Control Lett., vol. 19, no. 3 , pp. $177-185,1992$.

[16] M. Johansson and A. Rantzer, "Computation of piecewise quadratic Lyapunov functions for hybrid systems," IEEE Trans. Autom. Control, vol. 43, no. 4, pp. 555-559, Apr. 1998.

[17] T. A. Johansen, "Computation of Lyapunov functions for smooth nonlinear systems using convex optimization," Automatica, vol. 36, no. 11 , pp. 1617 - 1626, 2000.

[18] P. Giesl, Construction of Global Lyapunov Functions Using Radial Basis Functions, ser. Lecture Notes in Mathematics. Springer-Verlag Berlin Heidelberg, 2007, vol. 1904.

[19] A. Papachristodoulou and S. Prajna, "On the construction of Lyapunov functions using the sum of squares decomposition," in IEEE Conf. Decis. Control, vol. 3, Dec. 2002, pp. 3482-3487.

[20] W. Lohmiller and J.-J. E. Slotine, "On contraction analysis for nonlinear systems," Automatica, no. 6, pp. 683 - 696, 1998.

[21] E. M. Aylward, P. A. Parrilo, and J.-J. E. Slotine, "Stability and robustness analysis of nonlinear systems via contraction metrics and SOS programming," Automatica, vol. 44, no. 8, pp. $2163-2170,2008$.

[22] I. R. Manchester and J.-J. E. Slotine, "Control contraction metrics: Convex and intrinsic criteria for nonlinear feedback design," IEEE Trans. Autom. Control, vol. 62, no. 6, pp. 3046-3053, Jun. 2017.

[23] S. Singh, A. Majumdar, J.-J. E. Slotine, and M. Pavone, "Robust online motion planning via contraction theory and convex optimization," in IEEE Int. Conf. Robot. Automat., May 2017, pp. 5883-5890.

[24] W. Tan, "Nonlinear control analysis and synthesis using sum-ofsquares programming," Ph.D. dissertation, Univ. California, Berkeley, 2006.

[25] S. M. Richards, F. Berkenkamp, and A. Krause, "The Lyapunov neural network: Adaptive stability certification for safe learning of dynamical systems," in Conf. Robot Learn., vol. 87, Oct. 2018, pp. 466-476.

[26] Y.-C. Chang, N. Roohi, and S. Gao, "Neural Lyapunov control," in Adv. Neural Inf. Process. Syst., 2019, pp. 3245-3254.

[27] G. Shi et al., "Neural lander: Stable drone landing control using learned dynamics," in IEEE Int. Conf. Robot. Automat., May 2019.

[28] N. M. Boffi, S. Tu, N. Matni, J.-J. E. Slotine, and V. Sindhwani, "Learning stability certificates from data," in CoRL, Nov. 2020.

[29] H. K. Khalil, Nonlinear Systems, 3rd ed. Prentice-Hall, 2002.

[30] S. Hochreiter and J. Schmidhuber, "Long short-term memory," Neural Computation, vol. 9, no. 8, pp. 1735-1780, 1997.

[31] T. Miyato, T. Kataoka, M. Koyama, and Y. Yoshida, "Spectral normalization for generative adversarial networks," in Int. Conf. Learn. Representations, 2018.

[32] H. Tsukamoto and S.-J. Chung, "Imitation learning for robust and safe online motion planning: A contraction theory approach," Submitted to IEEE Robot. Automat. Lett., Feb. 2021.

[33] A. G. Barto, R. S. Sutton, and C. W. Anderson, "Neuronlike adaptive elements that can solve difficult learning control problems," IEEE Trans. Syst. Man Cybern., vol. SMC-13, no. 5, pp. 834-846, 1983.

[34] S. Diamond and S. Boyd, "CVXPY: A Python-embedded modeling language for convex optimization," J. Mach. Learn. Res., 2016.

[35] MOSEK ApS, MOSEK Optimizer API for Python 9.0.105, 2020.

[36] W. Li and E. Todorov, "Iterative linear quadratic regulator design for nonlinear biological movement systems," in Int. Conf. Inform. Control Automat. Robot., 2004, pp. 222-229. 